Wilfrid Laurier University

Scholars Commons @ Laurier

Physics and Computer Science Faculty

Publications

Physics and Computer Science

1999

\title{
Photon Statistics of a Single Atom Laser
}

\author{
Bobby Jones \\ Miami University -- Oxford, Ohio \\ Shohini Ghose \\ Wilfrid Laurier University, sghose@wlu.ca \\ James P. Clemens \\ Miami University -- Oxford, Ohio \\ Perry R. Rice \\ Miami University -- Oxford, Ohio \\ Leno M. Pedrotti \\ University of Dayton
}

Follow this and additional works at: https://scholars.wlu.ca/phys_faculty

\section{Recommended Citation}

Jones, Bobby; Ghose, Shohini; Clemens, James P.; Rice, Perry R.; and Pedrotti, Leno M., "Photon Statistics of a Single Atom Laser" (1999). Physics and Computer Science Faculty Publications. 67.

https://scholars.wlu.ca/phys_faculty/67

This Article is brought to you for free and open access by the Physics and Computer Science at Scholars Commons @ Laurier. It has been accepted for inclusion in Physics and Computer Science Faculty Publications by an authorized administrator of Scholars Commons @ Laurier. For more information, please contact scholarscommons@wlu.ca. 


\title{
Photon statistics of a single atom laser
}

\author{
Bobby Jones, Shohini Ghose, James P. Clemens, and Perry R. Rice \\ Department of Physics, Miami University, Oxford, Ohio 45056 \\ Leno M. Pedrotti \\ Department of Physics, University of Dayton, Dayton, Ohio 45469
}

(Received 3 February 1999)

\begin{abstract}
We consider a laser model consisting of a single four-level or three-level atom, an optical cavity, and an incoherent pump. Results for photon statistics for varying pump levels are obtained using a quantum trajectory algorithm. In particular, we calculate the mean photon number, Fano factor (which is the variance over the mean). We examine that the behavior of the single-atom device as $\beta$, the fraction of spontaneous emission into the lasing mode, is varied. Typical values considered for $\beta$ are $0.01<\beta<1$. . We find that for large enough $\beta$, lasing action, with properties similar to those predicted by semiclassical theories that factorize atom-field correlations and use a small-noise approximation, can occur. Squeezing can occur as $\beta$ is increased. There is no evidence of a sharp phase transition from weakly excited thermal light to coherent light at a particular pump power. This is consistent with work on many-atom lasers with $\beta$ values in the range considered here. As $\beta$ is increased, the output goes from quasithermal light to coherent and finally to squeezed light, progressing into a fully quantum-mechanical regime. We also consider the effects of cavity damping and spontaneous emission rates on these results. [S1050-2947(99)06510-5]

PACS number(s): 42.50.Ar, 42.50.Dv, 42.55.-f
\end{abstract}

\section{INTRODUCTION}

Recently it has become clear that cavity QED effects such as enhancement and suppression of spontaneous emission lasers might be used to favorably alter device properties [1]. Typically, the enhancement or suppression of spontaneous emission is accomplished through a reduction of the size of the device. In a cavity of transverse dimension less than half a wavelength, spontaneous emission to nonlasing modes is suppressed [2]. A decrease in cavity-mode volume increases coupling to the lasing mode. We refer to such devices as microcavity lasers. The reduction in size is also driven by a desire to have many such devices etched on a semiconducting wafer, in some sort of hybrid electro-optical computer.

A key parameter for microcavity lasers is $\beta$, the fraction of spontaneous emission into the lasing mode. In the limit that $\beta$ tends to unity, the gain medium is coupled only to one mode, the lasing mode. Hence, pump energy leaves the atomic system in the form of photons in the cavity mode, which then exit the cavity through an output coupler. The input-output curve is thus linear with no abrupt transition from a nonlasing to a lasing state. This behavior has led to proposals for "thresholdless" lasers [3], and a long discussion about the location (or even existence) of a lasing transition akin to that described by DiGiorgio and Scully for "ordinary" lasers [4] in these devices. Recent studies of the photon statistics of high $\beta$ devices have illuminated the difference between various regimes of operation for a microcavity laser $[5,6]$. This work has been mainly concerned with many-atom systems. In this paper we investigate a singleatom laser, and the effect of cavity QED conditions on such a device. We emphasize that these single-atom lasers differ from micromasers/microlasers where atoms in the excited state are deposited in the cavity in some manner and then exit the cavity at some later time. The devices we consider may correspond to trapped atoms in a cavity, or perhaps an exciton in a quantum dot. In the latter case, many-body effects must also be considered. The former case may be approximated by dropping cold atoms from a magneto-optical trap, where a cavity transit time is on the order of hundreds of lifetimes $[7,8]$.

A number of authors have shown that the light emitted by a single, pumped atom placed into an optical cavity should exhibit characteristics similar to that emitted by macroscopic lasers provided that Jaynes-Cummings coupling between the atom and the field is large enough, and that a single-atom laser may emit amplitude squeezed light $[9,10,11]$. We continue those investigations with a systematic description of the output properties of a single atom laser as a function of $\beta$. A quantum trajectory approach is used for numerical convenience. We begin with a brief review of the quantum trajectory method in Sec. II. Section III deals with the details of the four-level laser model, and results for the photon statistics are presented in Sec. IV. The three-level system and its photon statistics are discussed in Sec. V, and we conclude in Sec. VI.

\section{QUANTUM TRAJECTORY THEORY}

We outline below the general quantum trajectory method in the form developed by Carmichael [12]. Other formulations also exist $[13,14]$, but this one is most direct for our purposes. Our system is described by a master equation that can be written in the general Lindblad form

$$
\dot{\rho}=-i\left[H_{s}, \rho\right]+\sum_{j}\left(\hat{C}_{j} \rho \hat{C}_{j}^{+}-\frac{1}{2} \hat{C}_{j}^{+} \hat{C}_{j} \rho-\frac{1}{2} \rho \hat{C}_{j}^{+} \hat{C}_{j}\right),
$$

where the terms involving the $\hat{C}_{j}$ (which are sometimes called collapse operators) describe the system-reservoir cou- 
pling. Formally, the master equation can be written $\dot{\rho}=\mathbf{L} \rho$. The simulation equations can be generated by first identifying terms that can be written as commutators or anticommutators. These terms can be represented by a wave function undergoing a Schroedinger equation evolution using a nonHermitian Hamiltonian. The remaining terms identify the superoperator $\mathbf{S}$

$$
\mathbf{S} \rho=\sum_{j} \hat{C}_{j} \rho \hat{C}_{j}^{+}
$$

The parts of the master equation that can be written as commutators or anticommutators are then written as

$$
\begin{aligned}
(\mathbf{L}-\mathbf{S}) \rho & =-\frac{i}{\hbar}\left[H_{S}, \rho\right]+\left[H_{D}, \rho\right]_{+} \\
& =-\frac{i}{\hbar}\left[H_{S}, \rho\right]-\frac{i}{\hbar}\left[i \hbar H_{D}, \rho\right]^{*} \\
& =-\frac{i}{\hbar}\left[H_{S}-i \hbar H_{D}, \rho\right]^{*},
\end{aligned}
$$

where

$$
\begin{gathered}
H_{D}=\frac{1}{2} \hat{C}_{j}^{+} \hat{C}_{j}, \\
{[A, B]_{+}=A B+B A,} \\
{[A, B]^{*}=A B-B^{+} A^{+} .}
\end{gathered}
$$

A quantum trajectory is associated with a stochastic wave function $\left|\psi_{c}(t)\right\rangle$ that is conditioned upon a history of observations recorded by ideal detectors that are capable of detecting every photon emitted by the system. Now, $\rho(t)$ is related to $\left|\psi_{c}(t)\right\rangle$ by

$$
\rho(t)=\overline{\left|\psi_{c}(t)\right\rangle\left\langle\psi_{c}(t)\right|}
$$

Here, the overbar represents an ensemble average over many trajectories. The time evolution of $\left|\psi_{c}(t)\right\rangle$ determines the instantaneous rates of random photon detection at the ideal detectors,

$$
r_{j}(t)=\left\langle\psi_{c}(t)\left|\hat{C}_{J}^{+} \hat{C}_{j}\right| \psi_{c}(t)\right\rangle
$$

If no photon is detected, the conditioned wave function is propagated forward in time by a non-Hermitian Hamiltonian, $H=H_{S}+i \hbar H_{D}$, so that

$$
\frac{d}{d t}\left|\psi_{c}(t)\right\rangle=\frac{-i H}{\hbar}\left|\psi_{c}(t)\right\rangle .
$$

Since the evolution doesn't preserve the norm of $\left|\psi_{c}(t)\right\rangle$, we normalize the wave function at each step. The temporal evolution is performed on the computer in small time steps via $\left|\psi_{c}(t+\Delta t)\right\rangle=e^{-(i / \hbar) H \Delta t}\left|\psi_{c}(t)\right\rangle \approx[1-(i H \Delta t / \hbar)]\left|\psi_{c}(t)\right\rangle$.

The times at which the photon emissions occur is determined in Monte Carlo style using the rates $r_{j}(t)$. The probability of an event occurring in $(t, t+\Delta t)]$ is $r_{j}(t) \Delta t$. Each emission event entails a wave function collapse,

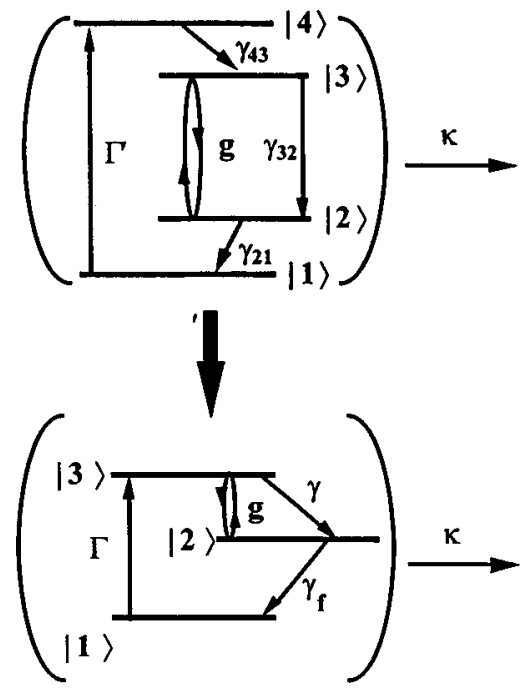

FIG. 1. Schematic diagram of a single four-level atom in a cavity with an incoherent pump, and level 4 adiabatically eliminated. For the four-level system, $\gamma_{i j}$ 's are spontaneous emission rates from level $i$ to $j$, and $\Gamma^{\prime}$ is a pump rate. For the three-level system $\Gamma$ is an effective pump rate, $\gamma$ is the spontaneous emission rate on the lasing transition, and $\gamma_{f}$ is the spontaneous emission rate from the lower lasing level. For both systems, $\kappa$ is the cavity decay rate and $g$ is the atom-field coupling strength.

$$
\left|\psi_{c}(t)\right\rangle \rightarrow \frac{\hat{C}_{j}\left|\psi_{c}(t)\right\rangle}{\sqrt{\left\langle\psi_{c}(t)\left|\hat{C}_{j}^{+} \hat{C}_{j}\right| \psi_{c}(t)\right\rangle}}
$$

representing the action of $\mathbf{S}$ and subsequent normalization. This unraveling of the master equation corresponds to direct detection of photons and is a natural one to use when one wishes to determine the photon statistics of the field. In our case we will have several collapse processes, and our wave function will be conditioned on various sets of perfect photodetectors, one detecting photons emitted from the cavity, one detecting fluorescence out the side of the cavity at the frequency of the laser transition, and so on. The connection between this unraveling and others based, for example, on homodyne detection is well elucidated in the article by Wiseman [13]. The approach discussed above can be generalized to absorption events in the case of an incoherent pumping mechanism, as we will use here.

\section{SINGLE FOUR-LEVEL-ATOM LASER}

In our model we adiabatically eliminate the upper atomic level by assuming that the transition rate from it to the upper state of the lasing transition is much larger than the other rates in the model. A schematic diagram of the system is shown in Fig. 1. The wave function is written using the atomic-state, cavity-photon-number-state basis. In our numerical work, the cavity-photon-number states are typically truncated at 100 . The atomic raising and lowering operators are denoted $\sigma_{i j}=|j\rangle\langle i|$. Thus, $\sigma_{12}$ is a raising operator and $\sigma_{21}$ is a lowering operator. The cavity field operators are the boson annihilation, $a$, and creation, $a^{\dagger}$, operators. A single cavity mode is resonant with the atomic lasing transition whose interaction is described by the rotating wave, dipole approximation, Jaynes-Cummings Hamiltonian 


$$
H_{s}=i \hbar g\left(a^{\dagger} \sigma_{32}-a \sigma_{23}\right) .
$$

We begin with the master equation in the Born-Markoff approximation,

$$
\begin{aligned}
\dot{\rho}= & \frac{-i}{\hbar}\left[H_{s}, \rho\right]+\kappa\left(2 a \rho a^{\dagger}-a^{\dagger} a \rho-\rho a^{\dagger} a\right)+\frac{\Gamma}{2}\left(2 \sigma_{13} \rho \sigma_{31}\right. \\
& \left.-\sigma_{31} \sigma_{13} \rho-\rho \sigma_{31} \sigma_{13}\right)+\frac{\gamma}{2}\left(2 \sigma_{32} \rho \sigma_{23}-\sigma_{23} \sigma_{32} \rho\right. \\
& \left.\left.-\rho \sigma_{23} \sigma_{32}\right)+\frac{\gamma_{f}}{2} 2 \sigma_{21} \rho \sigma_{12}-\sigma_{12} \sigma_{21} \rho-\rho \sigma_{12} \sigma_{21}\right),
\end{aligned}
$$

where $g$ is the Jaynes-Cummings dipole coupling rate to the cavity field, $\Gamma$ is the pump rate, $\gamma$ is the radiative decay rate for the $|3\rangle \rightarrow|2\rangle$ transition, $\gamma_{f}$ is the radiative decay rate for the $|2\rangle \rightarrow|1\rangle$ transition, and $2 \kappa$ is the cavity energy-loss rate. Usually we will consider $\gamma_{f}$ much larger than $\gamma$, and refer to $\gamma_{f}$ as the fast decay rate. The incoherent pump is modeled by coupling levels 1 and 4 to a heat reservoir at a large negative temperature [15].

In the quantum trajectory formalism, we will take the conditioned wave function to be

$$
\begin{aligned}
& \left|\psi_{c}(t)\right\rangle=\sum_{n=0}^{\infty} A_{1, n}(t) e^{-i E_{1, n} t}|1, n\rangle+A_{2, n}(t) e^{-i E_{2, n} t}|2, n\rangle \\
& +A_{3, n}(t) e^{-i E_{3, n} t}|3, n\rangle .
\end{aligned}
$$

Here, the $E_{i, n}=n \hbar \omega+E_{i}$ are the energies of the joint atomfield eigenstates in the absence of the interaction. Here, $i$ denotes the atomic state and $n$ denotes the photon number. The $A_{i, n}$ are the conditioned probability amplitudes associated with these states. The non-Hermitian Hamiltonian is

$$
\begin{aligned}
H_{D}= & \hbar(\omega-i \kappa) a^{\dagger} a+\hbar \omega \frac{\sigma_{z}}{2}+h \omega_{1} \Sigma+i \hbar g\left(a^{\dagger} \sigma_{32}-a \sigma_{23}\right) \\
& -i \hbar \frac{\gamma}{2} \sigma_{23} \sigma_{32}-i \hbar \frac{\gamma_{f}}{2} \sigma_{12} \sigma_{21}-i \hbar \frac{\Gamma}{2} \sigma_{31} \sigma_{13}
\end{aligned}
$$

where $\hbar \omega$ is the energy of a photon produced in the lasing transition $(3 \rightarrow 2)$ and $\sigma_{z}$ is the usual Pauli operator for the lasing transition. We have also defined $\omega_{1}=E_{1} / \hbar$ and $\Sigma$ $=|1\rangle\langle 1|$ as the energy and population operator of level 1 .

The collapse processes are chosen, in accordance with Eqs. (2) and (3), and master equation (10) to be

$$
\begin{aligned}
& \hat{C}_{1}=\sqrt{\gamma} \sigma_{32}, \\
& \hat{C}_{2}=\sqrt{\gamma_{f}} \sigma_{21}, \\
& \hat{C}_{3}=\sqrt{\Gamma} \sigma_{13}, \\
& \hat{C}_{4}=\sqrt{2 \kappa} a .
\end{aligned}
$$

These represent the following four processes: spontaneous emission on $|3\rangle$ to $|2\rangle$ to noncavity modes, spontaneous emission on $|2\rangle$ to $|1\rangle$ to all modes, incoherent pumping, and cavity decay. The corresponding probabilities of collapse in time $\Delta t$ are found using the rates of collapse given by Eq. (6) and the wave function Eq. (11) to be

$$
\begin{gathered}
P_{1}=\gamma \Delta t \sum_{n=0}^{\infty}\left|A_{3, n}\right|^{2}, \\
P_{2}=\gamma_{f} \Delta t \sum_{n=0}^{\infty}\left|A_{2, n}\right|^{2}, \\
P_{3}=\Gamma \Delta t \sum_{n=0}^{\infty}\left|A_{1, n}\right|^{2}, \\
P_{4}=2 \kappa \Delta t \sum_{n=0}^{\infty} n\left(\left|A_{1, n}\right|^{2}+\left|A_{2, n}\right|^{2}+\left|A_{3, n}\right|^{2}\right) .
\end{gathered}
$$

The non-Hermitian evolution of the conditioned probability amplitudes is given by

$$
\begin{gathered}
\dot{A}_{1, n}=-\left(\frac{\Gamma}{2}+n \kappa\right) A_{1, n}, \\
\dot{A}_{2, n+1}=-\left(\frac{\gamma_{f}}{2}+(n+1) \kappa\right) A_{2, n+1}+g \sqrt{n+1} A_{3, n}, \\
\dot{A}_{3, n}=-\left(\frac{\gamma}{2}+n \kappa\right) A_{3, n}-g \sqrt{n+1} A_{2, n+1} .
\end{gathered}
$$

Let us consider what happens when $\gamma_{f}$ is the fastest of all the rates $\left(\gamma_{f} \gg \gamma, g, \kappa, \Gamma\right)$. In this case the amplitude associated with the lower lasing state relaxes to steady state very rapidly so that Eq. (15b) can be solved in steady state and the result used in Eq. (15a). We find

$$
\dot{A}_{3, n}=-\left(\frac{\gamma}{2}+n \kappa\right) A_{3, n}-\frac{g^{2}}{\kappa(n+1)+\gamma_{f} / 2}(n+1) A_{1, n+1} .
$$

This form allows us to deduce the spontaneous emission factor $\beta$. With $n=0$, we may identify the first term as spontaneous emission into the nonlasing modes, and the second term as spontaneous emission into the lasing mode; hence, we have

$$
\beta=\frac{2 g^{2} / \gamma_{f}}{2 g^{2} / \gamma_{f}+\gamma / 2} .
$$

Note that we have ignored $\kappa$ compared to $\gamma_{f} / 2$ in arriving at this expression. This expression is consistent with a calculation of the spontaneous emission rate into the cavity mode using Fermi's golden rule; the transition rate is given by the square of the matrix element $(g)$ times the density of states [inverse bandwidth $=\left(\gamma_{f} / 2\right)^{-1}$ ]. This is the numerator of $\beta$, while the denominator is the total spontaneous emission rate $\left(2 g^{2} / \gamma_{f}+\gamma / 2\right)$, where the latter term is the spontaneous emission rate out the side of the cavity. Outside the goodcavity limit the spontaneous emission factor would take the form $\beta=\left[2 g^{2} /\left(\gamma_{f}+2 \kappa\right)\right] /\left[2 g^{2} /\left(\gamma_{f}+2 \kappa\right)+\gamma / 2\right]$.

We typically ran the code for 10000 atomic lifetimes, with a time step chosen as one hundredth of the inverse of 


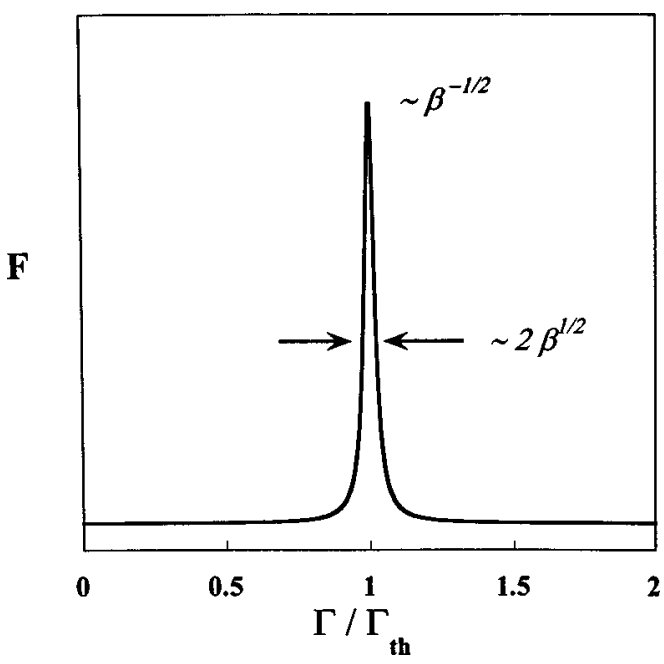

FIG. 2. Behavior of the Fano factor as a function of the pump for a macroscopic laser. Note that as $\beta$ increases the peak gets smaller and wider.

the fastest rate in the problem. A separate random number was used to determine whether one of the four collapses occurred in a time step $d t$. In the rare case that multiple collapses might occur in a given time step, we randomly pick one. There are other methods for dealing with this, but they all become equivalent for small time steps.

Note also that the Eqs. (15) are coupled only to each other. So during the non-Hermitian evolution, we need only propagate 3 quantities instead of $3^{*} n$. Collapses increase or lower the photon number index $n$. This is due to the incoherent nature of the pump and decay processes, which are collapse processes in this formalism.

\section{PHOTON STATISTICS OF THE FOUR-LEVEL LASER}

We calculate a number of physical quantities of interest including the average intracavity photon number,

$$
\langle n\rangle=\left\langle\psi_{c}\left|a^{\dagger} a\right| \psi_{c}\right\rangle,
$$

and the Fano factor $F$ (photon number variance/mean),

$$
F=\frac{\left\langle n^{2}\right\rangle-\langle n\rangle^{2}}{\langle n\rangle}=1+\langle n\rangle\left[g^{(2)}(0)-1\right] .
$$

Here, $g^{(2)}(0)=\left\langle a^{\dagger} a^{\dagger} a a\right\rangle /\left\langle a^{\dagger} a\right\rangle^{2}$ is the usual second-order intensity-correlation function. We examine these as a function of pump rate $\Gamma$ for different values of $\beta / \gamma, \kappa / \gamma$, and $\gamma_{f} / \gamma$. For purposes of comparison, Fig. 2 shows a typical Fano factor vs the pumping-rate plot for a conventional small $\beta$ laser. The large fluctuations at the threshold pump value $\left(\Gamma_{\text {th }}\right)$ are indicative of a well-defined phase transition. We begin by examining the average photon number versus pump rate in Figs. 3(a) and 3(b); the latter figure is an enlargement of the small pump regime of the former. In these figures we see that the photon number rises with the pump and then saturates at a very small value for large pump rates. This is true for smaller values of $\beta$ as well. As this is a single-atom device, it does no good to pump at a rate faster than the fastest decay rate, which acts as a bottleneck. Until the atom makes the transition from $|2\rangle$ to $|1\rangle$, it cannot be reexcited to
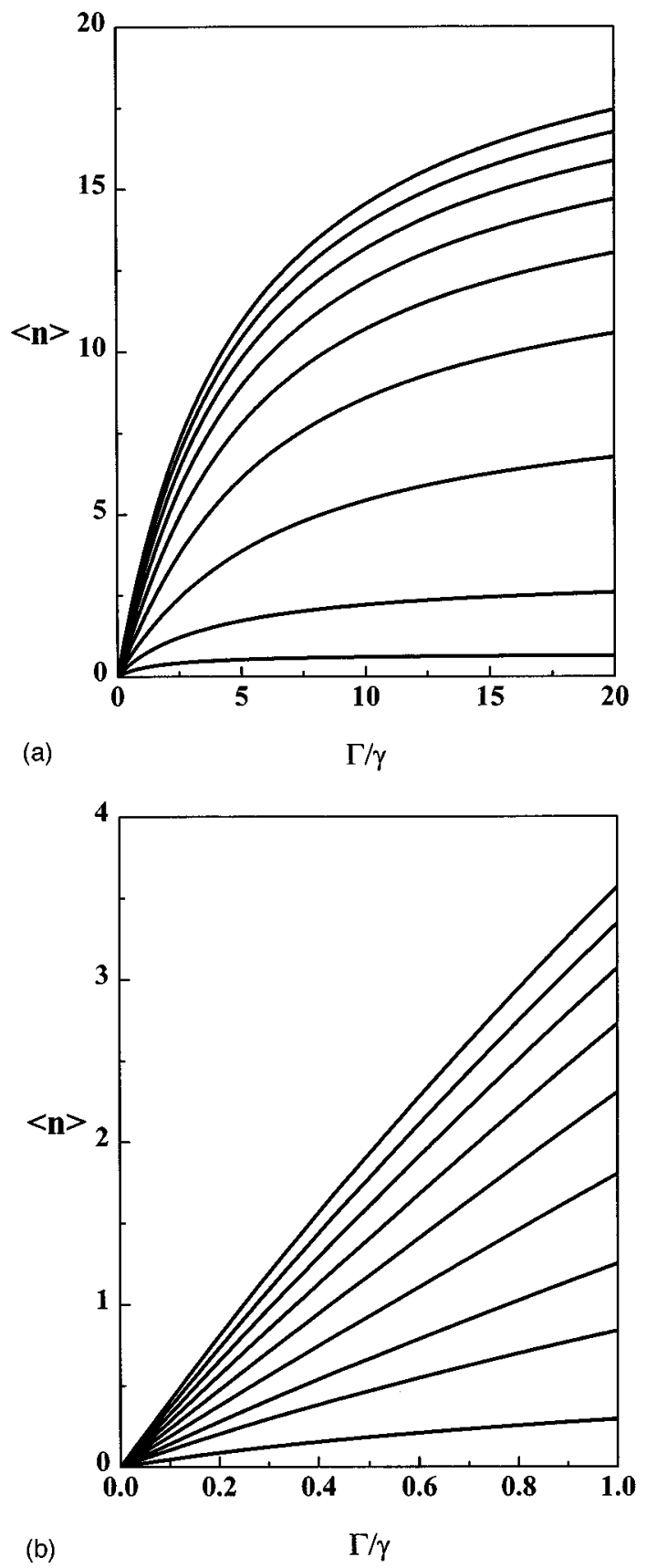

FIG. 3. (a) Mean photon number versus pump for various values of $\beta$ for large pumps. For all plots, $\gamma_{f} / \gamma=10.0$ and $\kappa / \gamma=0.1$. Curves are from bottom to top; $\beta=0.1, \beta=0.2, \beta=0.3, \beta=0.4$, $\beta=0.5, \beta=0.6, \beta=0.6, \beta=0.7, \beta=0.8, \beta=0.9$. (b) Mean photon number versus pump for various values of $\beta$ for small pumps. For all plots, $\gamma_{f} / \gamma=10.0$ and $\kappa / \gamma=0.1$. Curves are from bottom to top; $\beta=0.1, \beta=0.2, \beta=0.3, \beta=0.4, \beta=0.5, \beta=0.6, \beta=0.6, \beta$ $=0.7, \beta=0.8, \beta=0.9$.

level $|4\rangle$. For small pump values, the mean photon is proportional to the pump rate, independent of $\beta$.

We next consider the Fano factor as a measure of the fluctuations in the photon number in Fig. 4. For $\beta=0.3$, we see find $F=1$ for essentially zero pump, and then rises to some value and saturates. This is indicative of the behavior for smaller $\beta$ as well, although the asymptotic value for large pumps is smaller. As $\beta$ is increased to 0.5 we observe the Fano factor showing a peak and then dropping back to unity. 


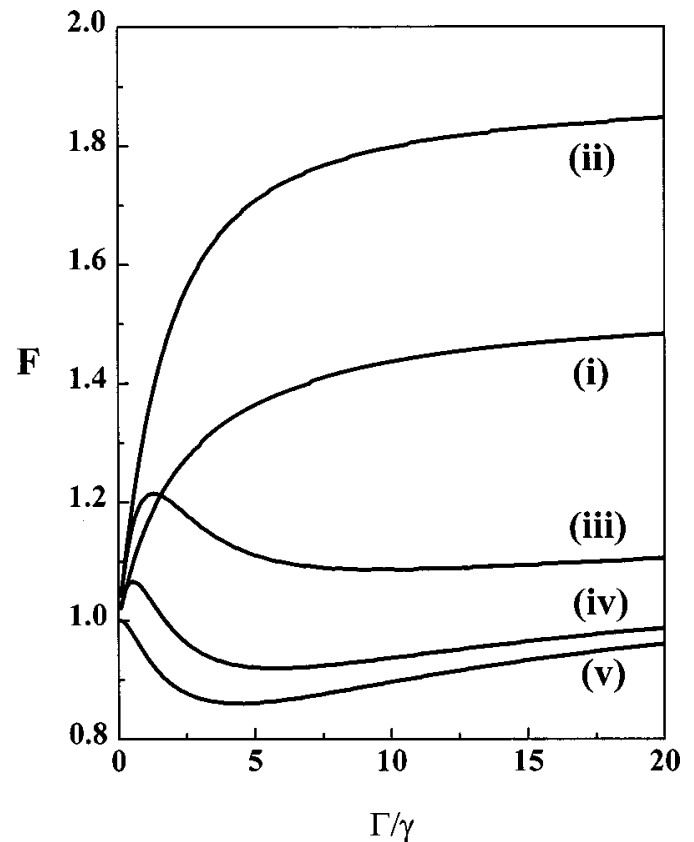

FIG. 4. Fano factor versus pump rate for various values of $\beta$. For all plots, $\gamma_{f} / \gamma=10.0$ and $\kappa / \gamma=0.1$. (i) $\beta=0.1$, (ii) $\beta=0.3$, (iii) $\beta=0.5$, (iv) $\beta=0.7$, and (v) $\beta=0.9$.

This small fluctuation peak is all that remains of the peak depicted in Fig. 2 for macroscopic lasers. Please note that the maximum value of the Fano factor occurs well after the mean photon number is greater than 1 . In most lasers, these features occur at the same pump rate. In fact, either criteria has been used to locate $\Gamma_{\text {th }}$ for macroscopic lasers. It has been proposed that the pump rate at which $\langle n\rangle=1$ be the threshold pump rate [16]. Clearly these criteria lead to different thresholds for the single-atom laser considered here. What is the correct criteria to use? When is this device a laser? We can determine no pump rate at which an infinitesimal change in pump switches the device from a nonlasing state to a laser. We see that different criterion for determining the threshold pump, which give the same result for small $\beta$ lasers, yield different values when applied to large $\beta$ lasers as discussed by Rice and Carmichael [5]. At high $\beta$ values, one is not in the thermodynamic limit and one does not expect a sharp threshold, no matter how many atoms form the gain medium. Here in the single-atom device we see a smeared out remnant of the phase transition predicted for many-atom lasers [5].

Increasing $\beta$ to around 0.7 leads to a very small peak in the Fano factor, and an asymptotic value less than one, indicating antibunching as first predicted by $\mathrm{Mu}$ and Savage [6]. For $\beta$ values greater than 0.8 or so, the Fano factor is 1 for small pumps and decreases to a value slightly above 0.85 . This photon antibunching or number squeezing is analogous to that observed by several groups [17-20] in many-atom lasers. In that work, the number squeezing was observed in a regime of strong pumping and fast decay rates connecting nonlasing levels. The maximal antibunching predicted here is similar to that predicted in the many-atom systems. This antibunching occurs due to an effective pump regularization, as the atom rarely lingers in a given state, but is excited and decays in a more regular fashion, giving rise to a rather regular sequence of photon emission [17-20]. As noted by $\mathrm{Mu}$

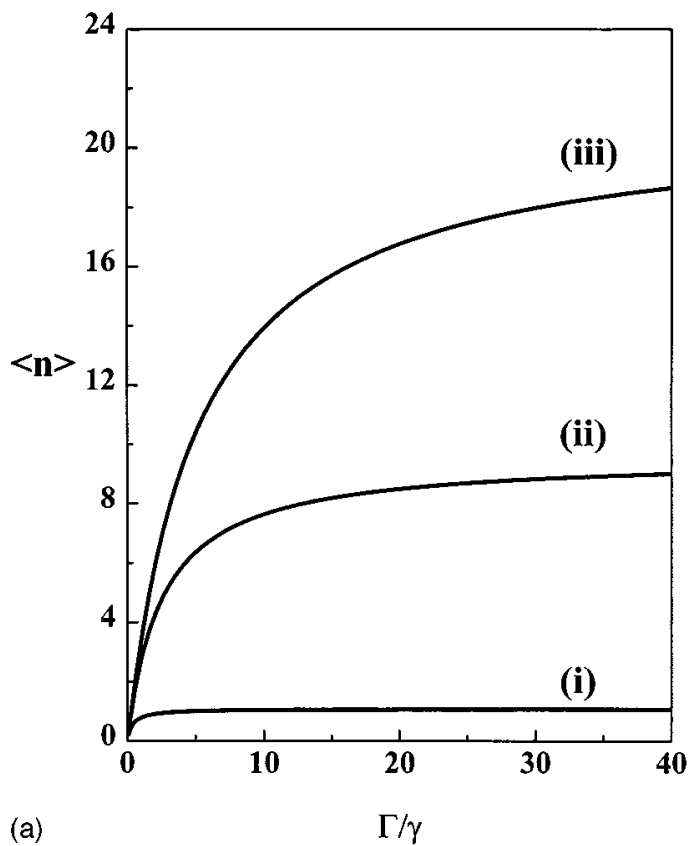

(a)

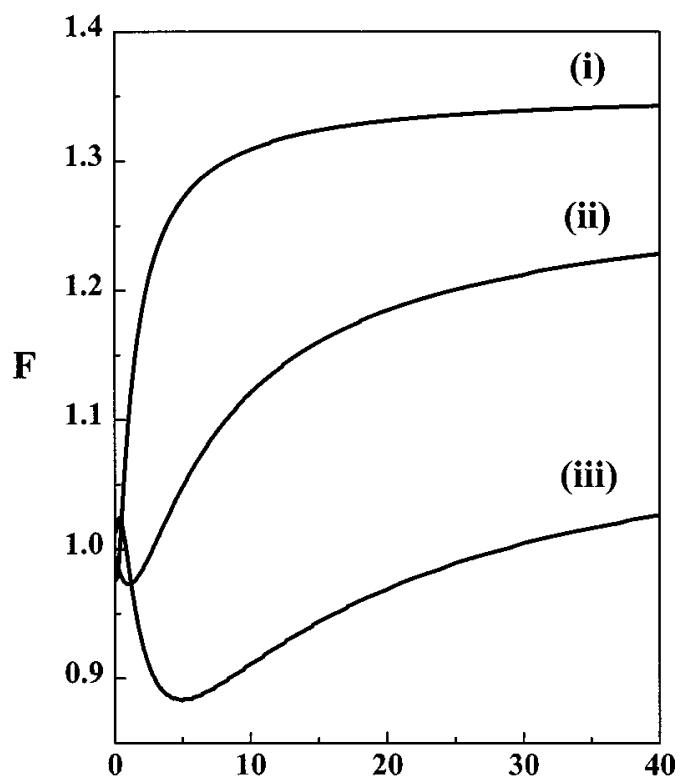

(b)

$\Gamma / \gamma$

FIG. 5. (a) Mean photon number vs pump rate with $\beta=0.8$ and $\kappa / \gamma=0.1$ for various $\gamma_{f}$. We have labeled curves by (i) $\gamma_{f} / \gamma$ $=1.0$, (ii) $\gamma_{f} / \gamma=5.0$, and (iii) $\gamma_{f} / \gamma=10.0$. (b) Fano factor vs pump rate with $\beta=0.8$ and $\kappa / \gamma=0.1$ for various $\gamma_{f}$. We have labeled curves by (i) $\gamma_{f} / \gamma=1.0$, (ii) $\gamma_{f} / \gamma=5.0$, and (iii) $\gamma_{f} / \gamma$ $=10.0$.

and Savage [9], this is essentially a single-atom effect, and persists in the limit of a one-atom laser.

We now examine the dependence of $\langle n\rangle$ and $F$ on $\gamma_{f}$, the transition rate out of the lower lasing level. In Fig. 5(a) we plot the mean photon number versus pump for a variety of values of $\gamma_{f}$ (with $\beta=0.8$ and $\kappa / \gamma=0.1$ ). As noted earlier, the mean photon number saturates at pump rates comparable to $\gamma_{f}$. In Fig. 5(b), we plot the Fano factor versus pump for a variety of values of $\gamma_{f} / \gamma$. We see that for small values of $\gamma_{f} / \gamma$, the Fano factor starts near unity and rises to an asymptotic value of 1.3 , indicating photon bunching. As 

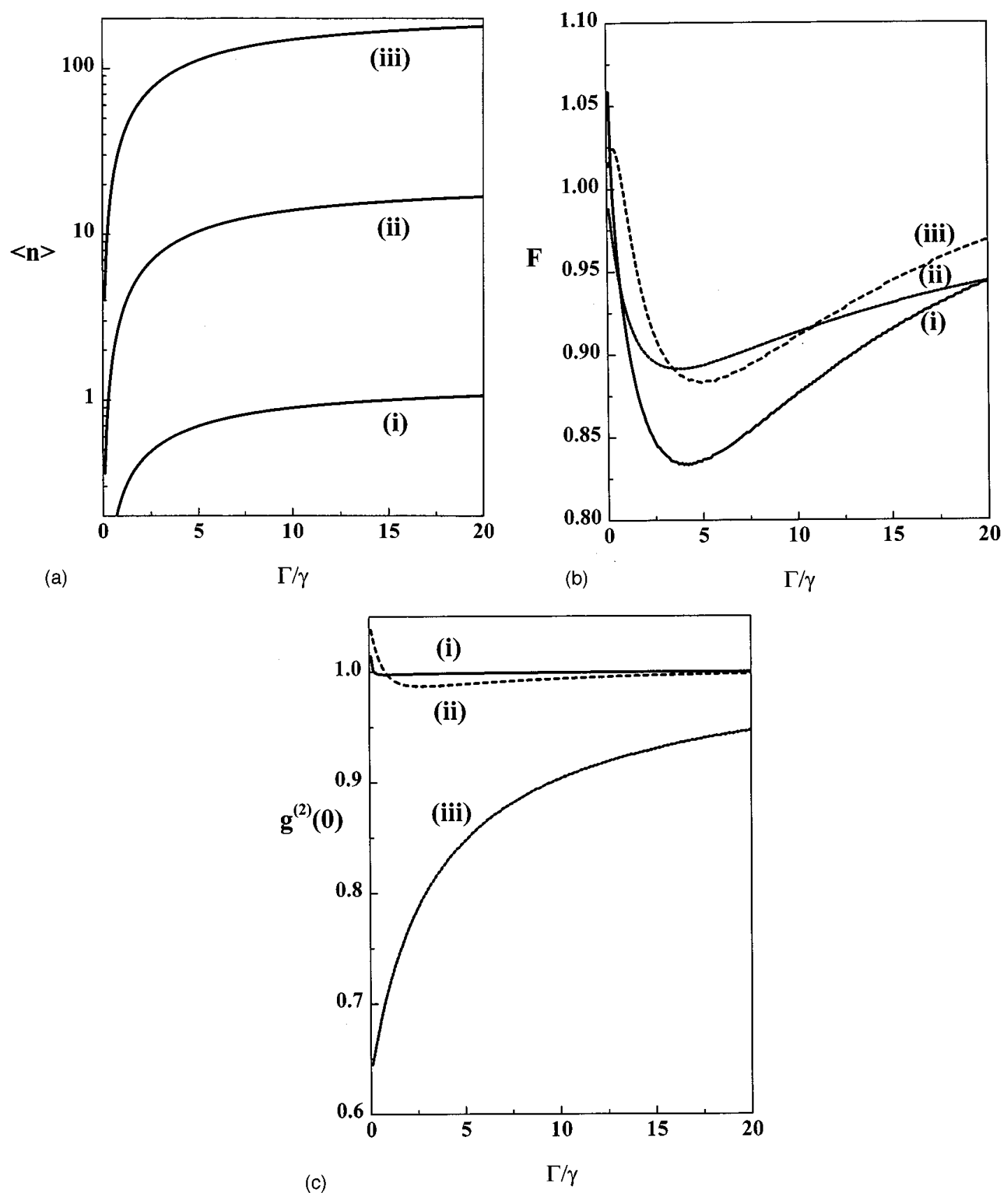

FIG. 6. (a) Mean photon number vs pump rate with $\beta=0.8$ and $\gamma_{f} / \gamma=10.0$ for various $\kappa$. We have labeled curves by (i) $\kappa / \gamma=1.0$, (ii) $\kappa / \gamma=0.1$, and (iii) $\kappa / \gamma=0.01$. (b) Fano factor vs pump rate with $\beta=0.8$ and $\gamma_{f} / \gamma=10.0$ for various $\kappa$. We have labeled curves by (i) $\kappa / \gamma=1.0$, (ii) $\kappa / \gamma=0.1$, and (iii) $\kappa / \gamma=0.01$. (c) $g^{(2)}(0)-1$ vs pump rate with $\beta=0.8$ and $\gamma_{f} / \gamma=10.0$ for various $\kappa$. We have labeled curves by (i) $\kappa / \gamma=1.0$, (ii) $\kappa / \gamma=0.1$, and (iii) $\kappa / \gamma=0.01$.

$\gamma_{f} / \gamma$ is increased, we see that the Fano factor decreases initially from 1, indicating antibunching, but then rises to an asymptotic value above 1 . Finally, as we make $\gamma_{f} / \gamma$ on the order of 10 or more, we see a small peak in the Fano factor for very small pumps, dropping to a value of about 0.85 , and then reaching 1 for large pumps. Here in the single-atom system, if $\gamma_{f}$ is small, there are essentially two independent Poisson processes that contribute to the noise, the fast transition and the pump. If $\gamma_{f}$ is large, then the atom very quickly relaxes to the ground state after emitting on the lasing transition, and so the only noise is the random nature of the pump, resulting in reduced fluctuations.

In Fig. 6(a), we plot the average photon number versus pump for a variety of values of $\kappa$. It is seen that decreasing $\kappa$ increases the intracavity photon number, $\langle n\rangle$ is proportional to $1 / \kappa$ as expected. In Fig. 6(b) we see that the Fano factor reaches a smaller minimal value for larger $\kappa$. In Fig. 6(c), however, we see that the amount of antibunching as measured by the second-order intensity-correlation function $g^{(2)}(0)-1=1 /\langle n\rangle(F-1)$ is actually greater for larger cavity decay rates. A reduction in antibunching is not surprising for a better cavity, as the field fluctuations are essentially averaged out. Photons leak out of the cavity in a Poisson process determined by the cavity decay rate for a good cavity. In a bad cavity, they are emitted and basically leave the cavity at the first encounter with the mirror. Hence, the bad- 


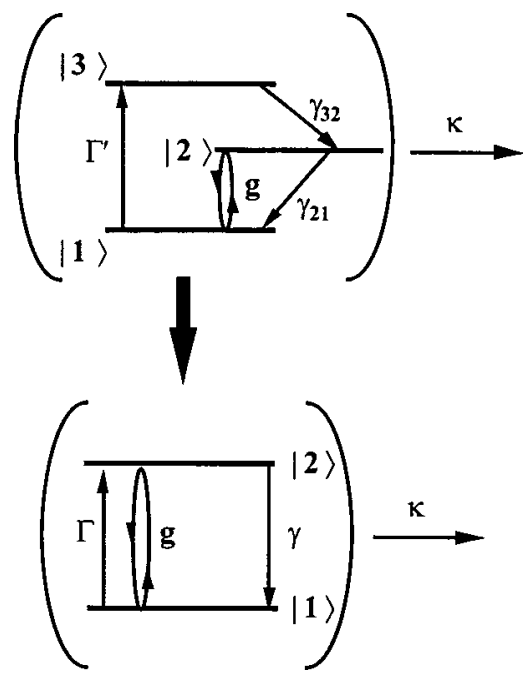

FIG. 7. Schematic diagram of single-atom three-level laser. For the three-level system, $\gamma_{i j}$ 's are spontaneous emission rates from level $i$ to $j, \Gamma^{\prime}$ is a pump rate. For the two-level system $\Gamma$ is an effective pump rate and $\gamma$ is the spontaneous emission rate on the lasing transition. For both systems, $\kappa$ is the cavity decay rate and $g$ is the atom-field coupling strength.

cavity system preserves the regular emission of the photons by the atom, leading to larger antibunching.

\section{SINGLE-ATOM THREE-LEVEL LASER}

The level structure for this system is depicted in Fig. 7. This system is described by the master equation

$$
\begin{aligned}
\dot{\rho}= & \frac{-i}{\hbar}\left[H_{s}, \rho\right]+\kappa\left(2 a \rho a^{\dagger}-a^{\dagger} a \rho-\rho a a^{\dagger}\right)+\frac{\gamma_{\downarrow}}{2}\left(2 \sigma_{-} \rho \sigma_{+}\right. \\
& \left.-\sigma_{+} \sigma_{-} \rho-\rho \sigma_{+} \sigma_{-}\right)+\frac{\gamma_{\uparrow}}{2}\left(2 \sigma_{+} \rho \sigma_{-}-\sigma_{-} \sigma_{+} \rho\right. \\
& \left.-\rho \sigma_{-} \sigma_{+}\right) .
\end{aligned}
$$

Here, $\kappa$ is the cavity decay rate as before, and $\sigma_{ \pm}$are the usual Pauli raising and lowering operators. The spontaneous emission rate out of the cavity is $\gamma_{\downarrow}$ and the incoherent pump rate is given by $\gamma_{\uparrow}$. In our quantum trajectory simulations, we use the following wave function:

$$
\left|\psi_{c}(t)\right\rangle=\sum_{n=0}^{\infty} B_{g, n}(t) e^{-i E_{g, n} t}|g, n\rangle+B_{e, n}(t) e^{-i E_{e, n} t}|e, n\rangle .
$$

Here, $B_{g, n}$ and $B_{e, n}$ are the conditioned probability amplitudes for the lower and upper lasing levels and $E_{g, n}$ and $E_{e, n}$ are the energies of these atom-field levels in the absence of an interaction. The non-Hermitan Hamiltonian is given by

$$
\begin{aligned}
H_{D}= & \hbar(\omega-i \kappa) a^{\dagger} a+\hbar \omega \frac{\sigma_{z}}{2}+i \hbar g\left(a^{\dagger} \sigma_{-}-a \sigma_{+}\right) \\
& -i \hbar \frac{\gamma_{\downarrow}}{2} \sigma_{+} \sigma_{-}-i \hbar \frac{\gamma_{\uparrow}}{2} \sigma_{-} \sigma_{+} .
\end{aligned}
$$

Here, $\hbar \omega$ is the energy difference between the lasing levels. There are now three collapse operators,

$$
\begin{aligned}
& \hat{C}_{1}=\sqrt{\gamma_{\downarrow}} \sigma_{-}, \\
& \hat{C}_{2}=\sqrt{\gamma_{\uparrow}} \sigma_{+}, \\
& \hat{C}_{3}=\sqrt{2 \kappa} a .
\end{aligned}
$$

These correspond to spontaneous emission events out the side of the cavity, incoherent pumping events, and photons exiting the output mirror. The associated probabilities for these events in a time $\Delta t$ are

$$
\begin{gathered}
P_{1}=\gamma_{\downarrow} \Delta t \sum_{n=0}^{\infty}\left|B_{e, n}\right|^{2}, \\
P_{2}=\gamma_{\uparrow} \Delta t \sum_{n=0}^{\infty}\left|B_{g, n}\right|^{2}, \\
P_{3}=2 \kappa \Delta t \sum_{n=0}^{\infty} n\left(\left|B_{g, n}\right|^{2}+\left|B_{e, n}\right|^{2}\right) .
\end{gathered}
$$

The probability amplitudes obey the following equations of motion:

$$
\begin{gathered}
\dot{B}_{g, n}=-\left(\frac{\gamma_{\uparrow}}{2}+n \kappa\right) B_{g, n}+g \sqrt{n} B_{e, n-1}, \\
\dot{B}_{e, n-1}=-\left(\frac{\gamma_{\downarrow}}{2}+n \kappa\right) B_{e, n-1}-g \sqrt{n} B_{g, n} .
\end{gathered}
$$

In this system, the spontaneous emission factor $\beta$ has the form

$$
\beta=\frac{2 g^{2} /\left(\gamma_{\downarrow}+\gamma_{\uparrow}\right)}{2 g^{2} /\left(\gamma_{\downarrow}+\gamma_{\uparrow}\right)+\gamma_{\downarrow} / 2} .
$$

Note that $\beta$ is pump-dependent. In the limit of small pumps and small coupling, this becomes $\beta=4 g^{2} / \gamma^{2}$. However, for large pumps the value tends towards $\beta=4 g^{2} / \gamma_{\downarrow} \gamma_{\uparrow}$, inversely proportional to the pump strength, and tending to zero in this limit. A plot of photon number versus pump is shown in Fig. 8(a). Here, we see $\langle n\rangle$ increases as the pump is increased, and then declines dramatically as the pump is further increased. This has been discussed by $\mathrm{Mu}$ and Savage [9] in terms of dephasing of the atomic dipole. Here we can see that it is a manifestation of the pump dependence of $\beta$ in the language of microlasers, and a reduction of spontaneous emission into the lasing mode. From the quantum trajectory point of view, it is obvious that a large incoherent pump rate is constantly "collapsing" the atom into the excited state, and hence no atomic polarization is ever generated, and hence no coupling to the lasing mode, in keeping with the original discussion of $\mathrm{Mu}$ and Savage [9]. A plot of the Fano factor, Fig. 8(b), indicates a fluctuation peak as the laser turns on, and then a broad fluctuation peak associated with turn off of the laser. Figure 8(c) exhibits the trapping of the atom in the excited state for strong incoherent pumps, which results in an effective decoupling from the field as previously 

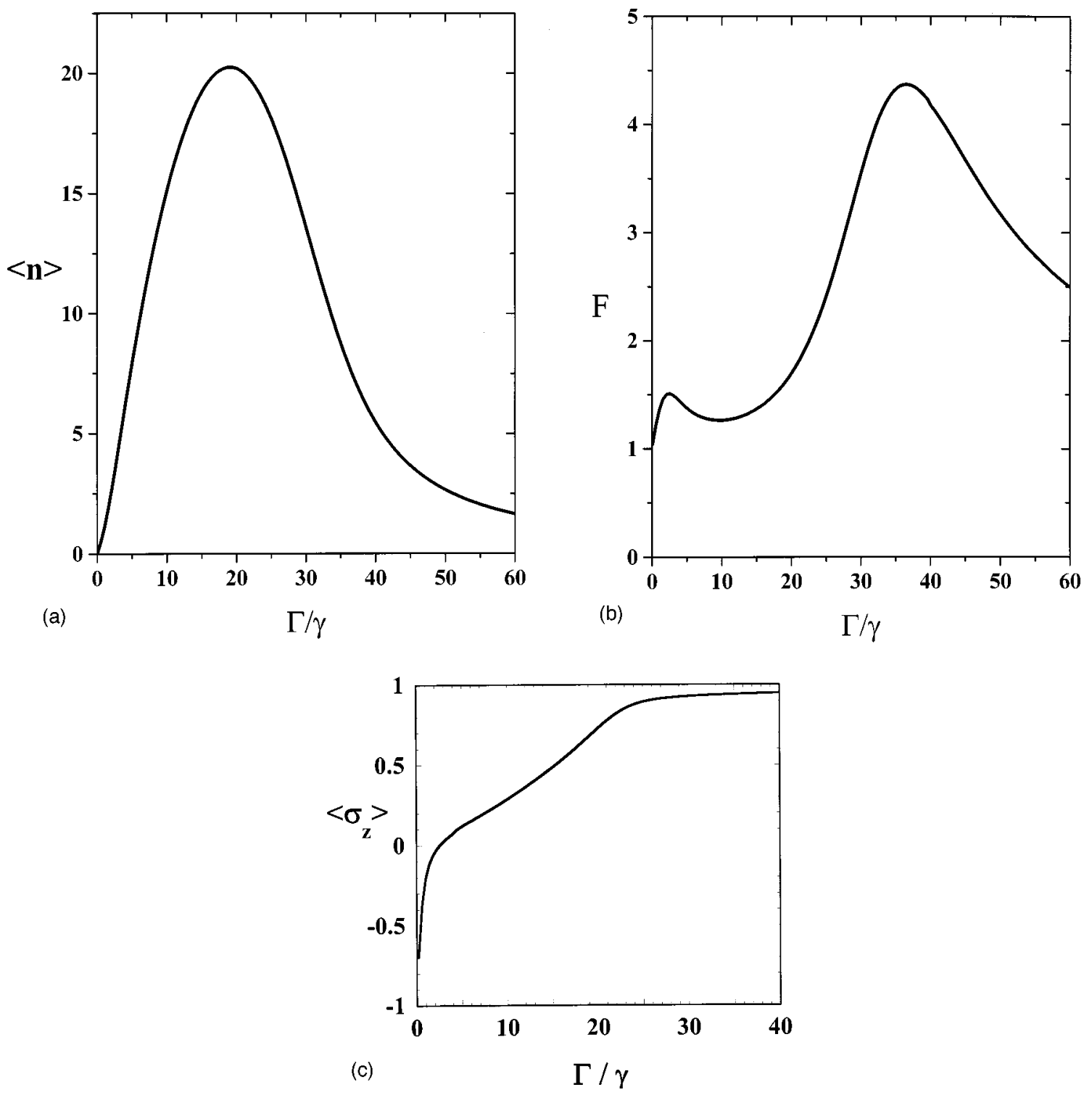

FIG. 8. (a) Mean photon number vs pump rate for $g / \gamma=1.414$ and $\kappa / \gamma=0.1$. (b) Fano factor vs pump rate for $g / \gamma=1.414$ and $\kappa / \gamma$ $=0.1$. (c) Population inversion vs pump rate for $g / \gamma=1.414$ and $\kappa / \gamma=0.1$.

discussed. This nonlinear dependence of laser intensity with pump rate for large pumps has also recently been investigated by Koganov and Shuker [21].

\section{CONCLUSIONS}

We have examined the behavior of a single-atom laser using both three- and four-level models, with particular emphasis on the behavior of the system as a function of the spontaneous emission factor $\beta$. In the four-level system, as $\beta$ is increased, we find the system changes from a superthermal emitter to a "smeared out" version of a semiclassical laser, until we reach a cavity-QED regime where amplitude squeezed light is emitted. There is no distinct laser threshold, and several of the usual criteria for threshold fall at very different pump values. For very small values of $\beta$, the device is essentially an light-emitting diode. For moderate values of $\beta$ near 0.2 , we find behavior that is qualitatively the same as in macroscopic lasers, with a change in slope of laser intensity with pump and a peak in the Fano factor. However, the pump rate at which the slope changes is distinct from the pump rate where the Fano factor peaks. For values of $\beta$ on the order of 0.5 or above, the essentially classical lasing behavior dissappears, and the device emits amplitude-squeezed (or number-squeezed) light. We have revisited the turning off of the three-level laser for large pumps, and interpreted this in terms of a pump-dependent $\beta$, as well as the trapping of the atomic population in the excited state killing off the induced dipole moment. This is consistent with previous discussions in terms of dipole dephasing.[9]

\section{ACKNOWLEDGMENTS}

We would like to thank Howard Carmichael, Michael Raymer, and Thomas Mossberg, for helpful discussions. 
[1] H. Yokoyama and S. D. Brorson, J. Appl. Phys. 66, 4801 (1988).

[2] Advances in Atomic and Molecular Physics, edited by P. Berman (Academic, San Diego, CA, 1994), Suppl. 2.

[3] Y. Yamamoto, S. Machida, and O. Nilsson, in Coherence, Amplification, and Quantum Effects in Semiconductor Lasers, edited by Y. Yamamoto (Wiley, New York, 1991), Chap. 11.

[4] V. DiGirogio and M. O. Scully, Phys. Rev. A 2, 1170 (1970).

[5] P. R. Rice and H. J. Carmichael, Phys. Rev. A 50, 4318 (1994).

[6] R. Jin, D. Boggavarpu, M. Sargent III, P. Meystre, H. M. Gibbs, and G. Khitrova, Phys. Rev. A 49, 4038 (1994).

[7] H. Mabuchi, Q. A. Turchette, M. S. Chapman, and H. J. Kimble, Opt. Lett. 21, 1393 (1996).

[8] J. Ng and L. A. Orozco (unpublished).

[9] Y. Mu and C. Savage, Phys. Rev. A 46, 5944 (1992).

[10] T. Pellizari and H. Ritsch, J. Mod. Opt. 41, 609 (1994).

[11] C. Ginzel, H.-J. Briegel, U. Martini, B. Englert, and A. Schenzle, Phys. Rev. A 48, 732 (1993).

[12] H. J. Carmichael, An Open Systems Approach To Quantum Optics (Springer-Verlag, Berlin, 1993); L. Tian and H. J. Car- michael, Phys. Rev. A 46, R6801 (1992).

[13] J. Dalibard, Y. Castin, and K. Molmer, Phys. Rev. Lett. 68, 580 (1992); R. Dum, P. Zoller, and H. Ritsch, Phys. Rev. A 45, 4879 (1992). The interested reader may also wish to consult Quantum Semiclassic. Opt. 8, 205 (1996), a special issue devoted to quantum trajectory theory.

[14] H. J. Wiseman, Ref. [13].

[15] H. Haken, in Light and Matter, edited by L. Genzel, Handbuch der Physik Vol. XXV/2c (Springer-Verlag, Berlin, 1970).

[16] G. Björk, A. Karlsson, and Y. Yamamoto, Phys. Rev. A 50, 1675 (1994).

[17] T. C. Ralph and C. M. Savage, Opt. Lett. 16, 1113 (1991); Phys. Rev. A 44, 7809 (1991).

[18] H. Ritsch and P. Zoller, Phys. Rev. A 45, 1881 (1992); H. Ritsch, P. Zoller, C. W. Gardiner, and D. F. Walls, ibid. 44, 3361 (1991).

[19] A. M. Khazanov, G. A. Koganov, and E. P. Gordov, Phys. Rev. A 42, 3065 (1990).

[20] D. L. Hart and T. A. B. Kennedy, Phys. Rev. A 44, 4572 (1991).

[21] G. Koganov and R. Shuker, Phys. Rev. A 58, 1559 (1998). 